\title{
Catheterization Related Complications Among Adults Male Patients with Urinary Retention in a Teaching Hospital
}

\author{
Muzzammil Abdullahi, Bashir Yunusa, Sharfuddeen Abbas Mashi, Sani Ali AJI, \\ Sani Usman Alhassan \\ Urology Unit, Department of Surgey, Bayero University / Aminu Kano Teaching Hospital, Kano, Nigeria
}

\section{Email address:}

saniaji2004@yahoo.com (S. A. AJI)

\section{To cite this article:}

Muzzammil Abdullahi, Bashir Yunusa, Sharfuddeen Abbas Mashi, Sani Ali AJI, Sani Usman Alhassan. Catheterization Related Complications Among Adults Male Patients with Urinary Retention in a Teaching Hospital. American Journal of Clinical and Experimental Medicine. Vol. 4, No. 4, 2016, pp. 109-111. doi: 10.11648/j.ajcem.20160404.14

Received: May 18, 2016; Accepted: May 30, 2016; Published: June 21, 2016

\begin{abstract}
Urethral catheterization is a common procedure in urologic practice. It is most commonly employed in relieving urinary retention. Various complications are associated with urethral catheterization and these depend on availability of expertise and strict observation of asepsis during the procedure. This study aimed at establishing the types and prevalence of complications associated with urethral catheterization among patients with urinary retention. It was a prospective study on 110 patients who presented in urinary retention and who were catheterize to relieve the retention. On presentation the patients were catheterize and urine was immediately taken for microscopy, culture and sensitivity. Patients with established UTI were excluded from subsequent investigation of catheter related UTI. Other complications of catheterization were also looked for and recorded. The patients age ranged from 17 to 100 years, with a mean age of $56 \pm 19.3$ SD years. Up to $74.5 \%$ of the patients in this study did not develop any of the complications. The most common complication was introduction of UTI in $17.3 \%$. This was followed by haematuria in $5.5 \%$ of the patients. Three patients $(2.7 \%)$ had their urethra injured and none had post obstructive diuresis. Catheterization related urinary tract infection is common despite observing asepsis in relieving urinary retention but other complications are not common.
\end{abstract}

Keywords: Catheterization, Complications, Urinary Tract Infection

\section{Introduction}

Urinary retention (UR) is defined as inability to empty the bladder despite being distended with urine [1]. It may be acute or chronic. In the acute form there is a sudden inability to pass urine despite the urge to micturate. This is usually associated with suprapubic pain. Chronic urinary retention (CUR) is an inability to empty the bladder completely during voiding which usually is not associated with suprapubic pain [1]. There may sometimes be an acute urinary retention (AUR) on a background of chronic urinary retention (acuteon-chronic urinary retention) [2].

Urinary retention is one of the common urologic emergencies worldwide $[2,3]$. The incidence is estimated to be 4.5-6.8 per 1000 men (aged 43-80 years) per year and this increases with increasing age in the USA [4]. Similarly, Hospital-based studies across West African Sub region have shown UR to be the commonest urologic emergency $[5,6,7]$. It is a common complication of urologic diseases such as Benign Prostatic Hyperplasia (BPH), urethral stricture, and bladder tumour.

The immediate management of UR is relieving the retention by urethral catheterization or suprapubic cystostomy (SPC). While in the acute form immediate decompression is allowed, in the chronic form, it is relieved gradually to prevent untoward effects of sudden decompression such as haematuria, post obstructive diuresis and hypotension $[1,8]$.

Several complications may be associated with relieving UR, this is more common in CUR where many health care providers have no adequate knowledge of the standard practice in relieving such UR. This could result in grave consequences sometimes leading to death from massive haemorrhage or post obstructive diuresis [9].

Suffice it to mention that in both AUR and CUR, 
complications from urethral catheterization could result, including injury to the urethra or bladder if the catheter balloon is inflated in the urethra or bladder neck and introduction of infection into the urinary tract which follows a breach in aseptic techniques [10].

\section{Patients and Method}

It was a hospital based prospective study of 110 adult male patients who consecutively presented in urinary retention (not relieved elsewhere) over one year. The patients were recruited through accident and emergency unit and the urology outpatient clinics of AKTH, Kano after obtaining informed consent from them or their care-givers and the information on the participants entered into the proforma.

On presentation, a brief history was taken and physical examination was done to establish the diagnosis of urinary retention and also to determine the type whether acute, chronic or acute-on-chronic. The clinical assessment was done also to detect conditions that may require resuscitation such as dehydration, anaemia, UTI and renal impairment. UR was relieved as part of the resuscitation. Urethral catheterization was initially used to relieve the retention in most patients, where it failed or was contraindicated then SPC was done. Volume of urine drained was recorded. In AUR the urine was drained at once while in CUR it was drained gradually over the following $24 \mathrm{hrs}$. All catheterizations were done in an aseptic setting by doctors in surgery department.

A detailed clinical assessment was then done to ascertain the possible cause (and predisposing/ precipitating factors) of the retention.

All the patients had their urine samples taken at catheterization for urinalysis and microscopy, culture and sensitivity. Full blood count (FBC), serum urea, electrolytes and creatinine $(\mathrm{U} / \mathrm{E} / \mathrm{Cr})$ and abdominal ultrasound were also done. Other investigations were done based on their indication. These investigations include retrograde urethrogram (RUG), micturating cystourethrogram (MCUG), Abdominal X-ray (KUB), PSA, prostate biopsy, cystoscopy/ urethrocystoscopy with or without biopsy. The results of the investigations were retrieved and recorded in the questionnaire during subsequent review in the ward or at urology outpatient clinic for the outpatients.

The patients who had UTI from the sample taken at presentation were excluded from subsequent evaluation this study. The evaluation was done during follow up; urine microscopy, culture and sensitivity was repeated after the first visit ( 2 weeks) and results recorded. In patients with other complications (anaemia and renal impairment), appropriate management was instituted. In both categories of patients, serum $\mathrm{U} / \mathrm{E} / \mathrm{Cr}$ and $\mathrm{PCV}$ were repeated to assess improvement from the baseline results.

Other complications related to catheterization such as urethral trauma, haematuria among others were also looked for and results recorded.

\section{Results}

The patients seen in this study were adult males of the ages ranged from 17 to 100 years with a mean age of $56 \pm 19.3 \mathrm{SD}$ years. The age distribution is shown in Figure 1.

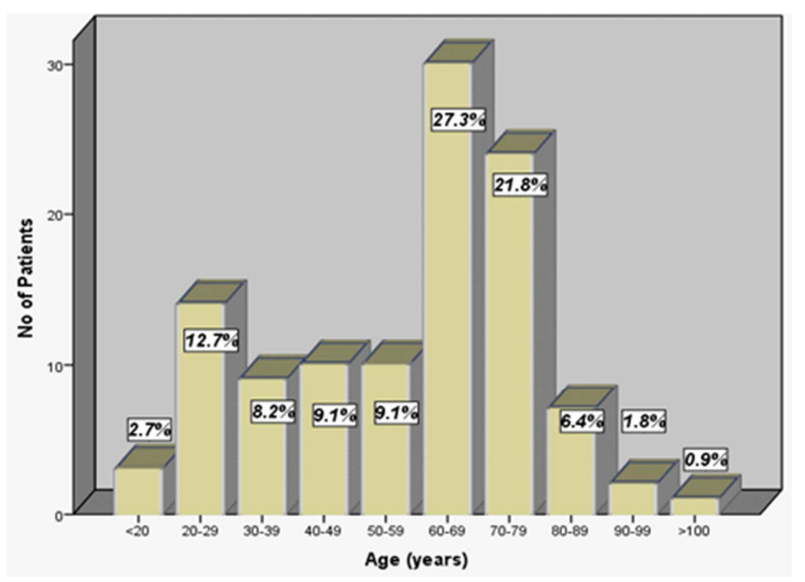

Figure 1. Age Distribution among the 110 Patients with Urinary Retention.

From this study, majority of the patients (53.4\%) were found to have AUR, 30.5\% had CUR while 16.1\% developed acute-on-chronic UR as shown in figure 2.

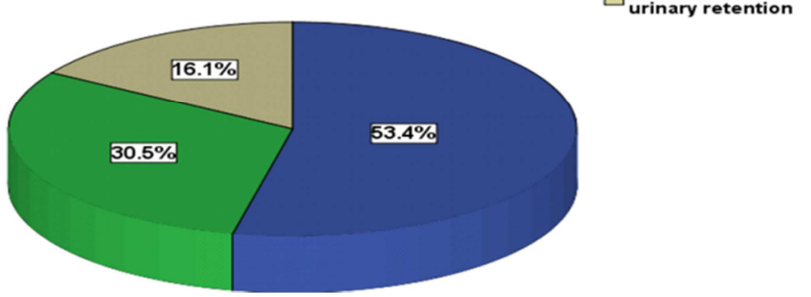

Figure 2. Showing the types of urinary retention.

Majority of the patients were catheterized with no complication. However, some patients had few complications as shown in Table 1.

Table 1. Complications of Relieving Urinary Retention with Catheterization $(n=110)$.

\begin{tabular}{lll}
\hline Complications & No of patients & Percent \\
\hline None & 82 & 74.5 \\
UTI & 19 & 17.3 \\
Haematuria & 6 & 5.5 \\
Urethral injury & 3 & 2.7 \\
Post obstructive diuresis & 0 & 0 \\
Total & 110 & 100 \\
\hline
\end{tabular}

The most common complication following catheterization noted in this study was UTI seen in 19 patients $(17.3 \%)$ followed by haematuria in 6 patients $(5.5 \%)$ and urethral injury in 3 patients $(2.7 \%)$. None had post obstructive diuresis.

Organisms cultured among the patients who developed UTI after catheterization are shown in table 2. 
Table 2. Microorgnisms cultured after catheterization.

\begin{tabular}{l|l|l}
\hline Organism & No of patients & percent \\
\hline Escherichia coli & 12 & 63.1 \\
Klebsiella spp & 4 & 21.1 \\
$\begin{array}{l}\text { Pseudomonas aeroginosa and } \\
\text { Klebsiella spp. }\end{array}$ & 3 & 15.8 \\
\hline
\end{tabular}

\section{Discussion}

Urinary retention is one of the common urologic emergencies in our environment.

Most patients who presented with UR were found to have AUR (53.4\%). Others were found to have CUR (30.5\%) and acute-on-chronic UR (16.1\%). Though there is paucity of reports comparing the incidence of the different types of urinary retention, many studies have shown that AUR is the most common urological emergency $[6,12]$.

The successful initial management of a patient with UR is by safe catheterization. This largely depends on the availability of trained personnel who should adopt appropriate techniques and take necessary precautions to prevent complications. However, some complications are known to be associated with catheterization. Up to $74.5 \%$ of the patients in this study did not develop any of the complications. The most common complication was introduction of UTI in $17.3 \%$. This was followed by haematuria in $5.5 \%$ of the patients. Three patients $(2.7 \%)$ had their urethra injured and none had post obstructive diuresis. The prevalence of UTI in this study was similar to the findings of Hans-Joerg Z, et al. who found $13 \%$ of their patients to have UTI at presentation [13]. Only $20.5 \%$ of the patients developed UTI in subsequent urine microscopy, culture and sensitivity.

The rate of urethral injury is low in this study. This is expected as the study was done in a tertiary hospital setting where catheterization is done generally under supervision of a urologist. None of the patients in this study developed post obstructive diuresis (polyuria of $\geq 3$ litres/day or persistent urinary output of $\geq 200 \mathrm{mls} / \mathrm{hr}$ ). This could be due to the low residual urine volume recorded in most of the patients at presentation $(753.7 \pm 164.8 \mathrm{SD} \mathrm{mls}, 1259 \pm 214.8 \mathrm{SD} \mathrm{mls}$ and 1937.2 \pm 276 SD mls for patients with AUR, CUR and acuteon-chronic UR respectively) which in turn may imply a moderate rise in intravesical pressure. The gradual decompression done in all the patients with CUR and acuteon-chronic UR could also explain the finding.

\section{Conclusion}

Urinary retention is common among the middle aged and the elderly; UTI is still a common complication of catheterization despite observing asepsis, and is predominantly caused by gram negative enterobacteriacae. However, the rate of urethral injury is low particularly in a tertiary health setting. Gradual decompression of the bladder in patients with CUR did not appear to prevent haematuria; however, post obstructive diuresis was not common.

\section{References}

[1] Klufio GD, Yeboah ED. Bladder, Urethra and Penis, Prostate. In Badoe EA, Archampong EQ, da Rocha Afodu JT (Eds). Principles and practice of surgery including pathology in the tropics, $4^{\text {th }}$ edition. Accra: Ghana publishing company. 2010: 881-883, 914-915, and 920-952.

[2] Odunayo K, Mark J S. Management of Acute and Chronic Retention in Men. European urology supplements. 2009; 8: 523-529.

[3] John MF, Francois D, Kamel A, et al. Management of Acute Urinary Retention: A Worldwide Survey of 6074 Men with Benign Prostatic Hyperplasia. BJU International. 2011; 109: $88-95$.

[4] Brian A, Selius DO, Rajesh S. Urinary retention in Adults: Diagnosis and Initial Management. Am Fam physician. 2008; 77: 643-650.

[5] Mbibu NH, Nwofor AME, Khalid L. Spectrum of Urologic Disease in the West African Sub region. Annals of African Medicine. 2002; 1: 44-52.

[6] Fall B, Diao B, Fall PA, et al. Urological Emergencies at the Dakar University Teaching Hospital: Epidemiological, Clinical and Therapeutic Features. Journal de L'Association Francaise D'urologie et de la Societe Francaise D'urologie. 2008; 18: 650-653.

[7] Patterson JM, Chapple CR. Failure of Urinary Drainage: Lower Tract. In Markus H, Santucci RA (Eds). Emergencies in Urology, $1^{\text {st }}$ edition. Berlin: Springer publishers. 2007; 118131.

[8] Michael T. Urinary Retention. In Macfarlane, Michael T (Eds). The Urology, $4^{\text {th }}$ edition. 2006; 1-6.

[9] Stephen OI, Aderinsola AO, Taiwo OO, et al. The burden of prolonged indwelling catheter after acute urinary retention in Ikeja - Lagos, Nigeria. BMC urol. 2007; 7: 1-4.

[10] Thomas K, Oades G, Taylor-Hay C, et al. Acute Urinary Retention: What Is the Impact on Patients' Quality of Life? BJU International. 2005; 95: 72-76.

[11] Neal DE. The urinary bladder. In Williams NS, Bulstrode CJK, O'Connell, PR (Eds). Bailey and Loves short practice of surgery, $25^{\text {th }}$ edition. London: Edward Arnold Publishers. $2008 ; 1314-1342$.

[12] Djones DA, George NJR. Interactive Obstructive Uropathy in Man. British Journal of Urology. 1992; 69: 337-345.

[13] Hans-Joerg Z., Michael AD, Wallace KS. Predictors of successful trial without catheter in acute urinary retention. British Journal of Medical and Surgical Urology. 2010; 3: 510. 